Hanley TH, Gallacher BJ, Grigg HTD. On the Exploitation of Mode Localization in Surface Acoustic Wave MEMS. Mechanical Systems and Signal Processing 2016

DOI: http://dx.doi.org/10.1016/i.ymssp.2016.07.018

Copyright:

(C) 2016. This manuscript version is made available under the CC-BY-NC-ND 4.0 license

Date deposited:

$12 / 08 / 2016$

Embargo release date:

05 August 2017

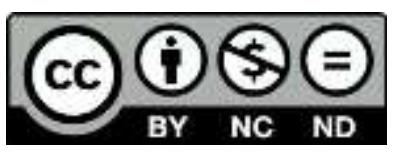

This work is licensed under a

Creative Commons Attribution-NonCommercial-NoDerivatives 4.0 International licence 


\title{
On The Exploitation of Mode Localization in Surface
}

\section{Acoustic Wave MEMS}

\author{
T. H. Hanley ${ }^{*}$, B. J. Gallacher ${ }^{1}$, H. T. D. Grigg ${ }^{1}$ \\ ${ }^{1}$ School of Mechanical and Systems Engineering, Newcastle University, \\ Newcastle Upon Tyne, NE1 7RU \\ * Corresponding author. Tel.: +44-07507-652110. \\ E-mail address: t.h.hanley@ newcastle.ac.uk.
}

\begin{abstract}
Mode localization sensing has been recently introduced as an alternative resonant sensing protocol. It has been shown to exhibit several advantages over other resonant methods, in particular a potential for higher sensitivity and rejection of common mode noise. This paper expounds the principles of utilising surface acoustic waves (SAW) to create a mode localization sensor. A generalised geometry consisting of a pair of coupled resonant cavities is introduced and an analytical solution found for the displacement fields within the cavities. The solution is achieved by coupling the internal cavity solutions using a ray tracing method. The results of the analytical solution are compared to a numerical solution found using commercial finite element method (FEM) software; exact agreement is found between the two solutions. The insight gained from the analytical model enables the determination of critical design parameters. A brief analysis is presented showing analogous operation to previous examples of mode localization sensors. The sensitivity of the device is shown to depend nonlinearly on the number of periods in the array coupling the two cavities.
\end{abstract}

Keywords: SAW; Mode localization; MEMS; micro-electromechanical systems; surface acoustic wave; sensor

\section{Introduction}

\subsection{Mode Localization Sensing}

Mode localization refers to the spatial trapping of energy in a coupled oscillatory system that occurs when a disorder is introduced into a previously ordered system. In the structures of interest in this article, this is manifested as a dramatic change in the mode shapes. This was first suggested as a novel sense protocol in references $[1,2]$ 
and coined 'mode localization sensing'. The sense mechanism was initially proposed as an alternative to measuring frequency-shift, and therefore this method has often been used as a benchmark for comparison [2-5].

Mode localization sensors have been approached primarily in the case of two weaklycoupled symmetrical oscillators [2-5]. They exploit the large change in the eigenvector that occurs when the symmetry is broken. A simplified model of a typical mass sensor utilising this protocol is presented in figure 1 . The identical resonators are coupled by the weak spring of strength $k_{c}$, producing a 2 degree of freedom (DOF) resonant system. In the case when $\Delta m=0$ the system exhibits both in-phase and out-of-phase modes with equal distribution of displacement amplitude. As is commonly known, the in-phase mode is the fundamental and the out-of-phase occurs at a higher frequency. When $\Delta m \neq 0$ the modal amplitude distribution is weighted unevenly, favouring the higher or lower mass DOF, in the in-phase and out-of-phase modes respectively. That is, if the system is vibrating in its fundamental mode, and a mass is added to one of the DOFs, this DOF will experience a much larger displacement relative to the other. In the second mode however, the un-laden mass will exhibit the larger relative displacement. The sensors are proposed to operate in one mode and use the ratio of the amplitude of the DOFs to detect the size of the added mass.
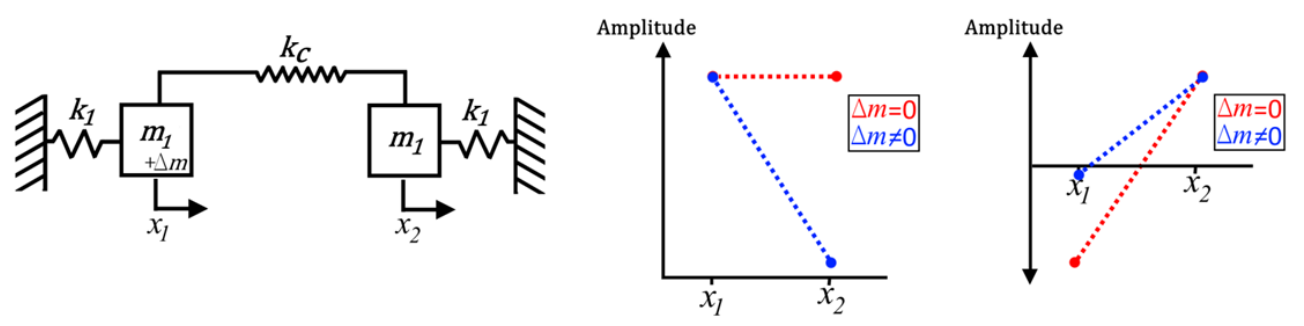

Figure 1: Typical example of a mass sensor using the mode localization sense protocol

\subsection{SAW Mode Localization Sensors}

The current work extends this theory to encompass the use of periodic structures to couple the resonators. This is realised through the use of a SAW architecture. The use of SAW presents many advantages when compared to the alternative of constructing periodic arrays of suspended MEMS. The SAW devices require a simple and inherently robust architecture; there are no deep cavity etches or slender beams. The manufacturing process is well established and SAW resonators are commonplace in 
microelectronics. The use of SAW was introduced in micro-scale electronic filters as a low noise alternative to lumped-parameter and bulk acoustic wave filters [6]. Therefore, the use of SAW presents a favourable platform to introduce a novel sense protocol, with the potential of yielding high signal-to-noise ratio devices. The authors introduced the concept of a SAW mode localization sensor in reference [7] and to their knowledge this is the first example available in the literature.

The proposed configuration consists of two cavities, coupled by an N-period reflective array. The arrays are constructed from a patterned metal film deposited on a piezoelectric substrate. In the general case, excitation can occur in either cavity, or both cavities. Regardless of the excitation method, one cavity is deemed the reference and the other the sense cavity. The coupling strength between the two cavities is governed by the shared periodic reflector, deemed the 'coupling array'. The basic arrangement is depicted in figure 2.

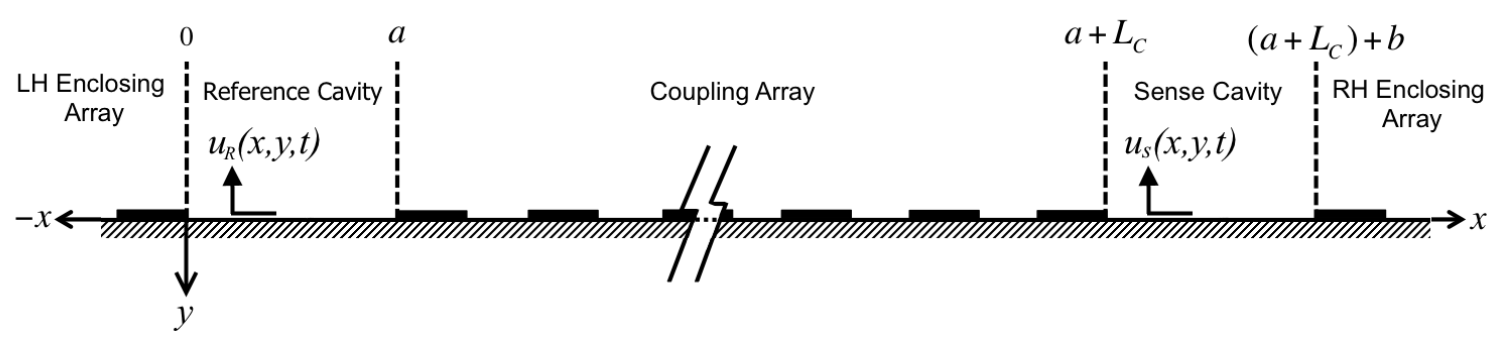

Figure 2: Device layout schematic

The output of the device is the ratio of the sense cavity displacement amplitude to the reference cavity displacement amplitude. The theory of operation is directly analogous to the mode localization sensors described in section 1.1: the output responds in proportion to a break in the symmetry of the structure, in this case, a perturbation of the wave-speed of the sense cavity. The output response is proportion to both the perturbation size and the coupling strength.

The main body of this paper is presented in section 2, the premises and assumptions are outlined prior to the presentation of the full derivation of the analytical model. Section 2.2 outlines the geometry and boundary conditions employed in the finite element model and section 2.3 contrasts the two models seeking validation of the analytical model. A brief analysis is presented in section 3, in which the frequency response of the device is discussed using the results of section 2. In addition, a 
qualitative overview of the envisioned device operation is described and finally conclusions are drawn.

\section{Modelling}

\subsection{Derivation of the analytical model}

Within this section, general expressions will be derived for the displacement fields within each cavity, as a function of the device parameters. It can be seen in fig. 2 that the device has been divided into five sections. The solutions within each section will be gained separately and then combined using a ray-tracing method. The reflection and transmission properties of each array are independent of one another with the exception of the coupling array. It is asserted that the proportion of reflection and transmission across the array is not dependent on the direction of wave travel, due to the symmetry of the coupling array.

The derivation of the model will follow a similar procedure to that conducted in reference [8], with the added complication of the transmission of waves between the two cavities. The reference and sense cavities will be considered separately to begin with, and their connection through the coupling array modelled using the transfer matrix approach as described in references [9-11].

The two-dimensional elastodynamic equations govern the wave motion in every section. The solutions sought are the steady-state Rayleigh wave solutions, assuming a harmonic solution at the forcing frequency. The forced solution has been presented in references [8] and [12]. The free Rayleigh wave solutions are well known, and presented in references [12] and [13]. However, the transfer matrix method applies rigorously only to one-dimensional solutions [14]. As the majority of the displacement field is confined to the surface, a one-dimensional approximate solution has been shown to provide accurate results [11]. The depth behaviour, $\xi_{j}(y)$, is found from the free wave solutions in the plated and un-plated substrate and integrated in the course of the derivation. The obvious limitation of this is that bulk waves generated from the forcing, or scattered from the discontinuities, are neglected. However, for plated regions where the plate thickness is small in comparison to the incident wavelength, the bulk scattering is minimal. The one-dimensional approximation is given in equation 1 . 


$$
u_{\mathrm{j}}(x, y, t)=\xi_{j}(y) \psi(x) \mathrm{e}^{-\mathrm{i} \Omega \mathrm{t}}
$$

Where $j=x$ or $y$ and $\psi(x)$ defines the $x$-dependence of the wave amplitude at the surface. This is the precise variable of interest in the current problem and thus the derivation will be conducted in a single dimension, solving for $\psi(x)$ in both cavities.

To simplify and approximate the forcing, due to an interdigitated transducer, the excitation will be represented as a dipole acoustic source. The displacement fields in a forced $\psi_{F}(x)$ and un-forced $\psi_{U F}(x)$ cavity, neglecting reflections, are now defined in equations 2-4. The convention that the physical wave is the real part of the expression is adopted and $x_{F}$ is the location of the source.

$$
\begin{aligned}
& \psi_{F}(x)_{x<x_{F}}=F_{L} e^{-i k_{\alpha} x} \\
& \psi_{F}(x)_{x>x_{F}}=F_{R} e^{i k_{\alpha} x} \\
& \psi_{U F}(x)=\mathrm{A} e^{i k_{\alpha} x}+\mathrm{B} e^{-i k_{\alpha} x}
\end{aligned}
$$

It can be seen that the field within the sense and forcing cavities will be described by the sum of a leftwards and a rightwards propagating wave, $e^{-i k_{\alpha} x}$ and $e^{i k_{\alpha} x}$ respectively, the amplitudes of which are to be determined. This is achieved by using a ray-tracing method and consideration of the physics of the problem.

Representing each array of reflectors as a single boundary, it can be seen that there are three fundamental paths that a wave can traverse before arriving back at its starting point. These paths are (a) internal reflection within the reference cavity, (b) internal reflection traversing both cavities and (c) internal reflection within the sense cavity. These are depicted in figure 3. Considering a wave propagating away from any boundary, any future wave originating at the same boundary due to that wave, is made up of the product of the original wave and some combination of these paths.

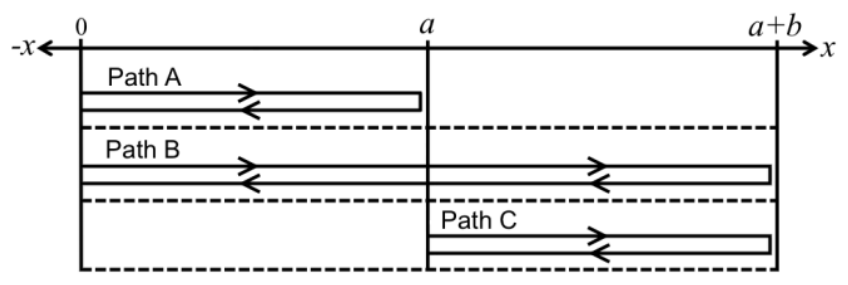

Figure 3 Loci of the fundamental reflection paths 
Each path is represented mathematically by a phase change due to distance travelled, and a complex amplitude coefficient due to the partial reflections and transmissions. The expressions for the paths are given in equations 5-7. Each path expression will be denoted as $\chi_{\alpha}$.

$$
\begin{aligned}
& \text { Path } A=\chi_{A}=R_{R} R_{C} e^{i 2 k_{0} a} \\
& \text { Path } B=\chi_{B}=R_{S} T_{C}^{2} R_{R} e^{i 2 k_{o}(a+\eta b)} \\
& \text { Path } C=\chi_{C}=R_{S} R_{C} e^{i 2 \eta k_{o} b}
\end{aligned}
$$

Where $R_{R}$ and $R_{S}$ are the reflection coefficients for the enclosing arrays of the reference and sense cavities respectively. $T_{C}$ and $R_{C}$ are the transmission and reflection coefficients of the coupling array. $k_{0}$ is the wavenumber within the reference cavity and $\eta=k_{S E N S E} / k_{0}$.

The solution within each cavity can therefore be said to be some product of the initial waves at each of its boundaries and a combination of the path expressions. The initial waves are defined as the excited or reflected waves that have not yet traversed one full fundamental path. Three path expression combinations (PECs) can be defined. These represent every possible combination of routes an initial wave can take as it is repeatedly internally reflected and transmitted between the two cavities. These take the forms shown in equations 8-10.

$$
\begin{aligned}
& \Gamma=\sum_{i=0}^{\infty} \sum_{j=0}^{\infty} \sum_{k=0}^{\infty}\left(\frac{(i+j) !}{i ! j !}\right)\left(\frac{(j+k) !}{j ! k !}\right) \chi_{A}{ }^{i} \chi_{B}{ }^{j} \chi_{C}{ }^{k} \\
& \Gamma_{R}=\sum_{i=0}^{\infty} \sum_{j=0}^{\infty} \sum_{k=0}^{\infty}\left(\frac{(i+j) !}{i ! j !}\right)\left(\frac{(j+k) !}{j ! k !}-\frac{(j+k-1) !}{j !(k-1) !}\right) \chi_{A}{ }^{i} \chi_{B}{ }^{j} \chi_{C}{ }^{k} \\
& \Gamma_{S}=\sum_{i=0}^{\infty} \sum_{j=0}^{\infty} \sum_{k=0}^{\infty}\left(\frac{(i+j) !}{i ! j !}-\frac{(i+j-1) !}{i !(j-1) !}\right)\left(\frac{(j+k) !}{j ! k !}\right) \chi_{A}{ }^{i} \chi_{B}{ }^{j} \chi_{C}{ }^{k}
\end{aligned}
$$

Equation 8 is the sum of every possible combination of the three fundamental paths. Equation 9 is the same as 8 less all combinations in which path $\chi_{C}$ occurs before at least one incidence of path $\chi_{B}$. This expression is necessary for waves that originate 
from excitation within the reference cavity. The initial waves cannot traverse path $\chi_{C}$ without this path occurring after path $\chi_{B}$ has begun. The same reasoning is used for equation 10, however, in relation to initial waves due to an excitation in the sense cavity.

It is of note that the path expressions have all been placed within an infinite sum. This is due to the fact that, neglecting damping effects, the waves will repeat each reflection and transmission pattern indefinitely. It is necessary to simplify the expressions so that they can be analytically inspected and numerically evaluated. The summations are combinations of infinite arithmetico-geometric progressions. These can be evaluated using equation 11 .

$$
\sum_{n=0}^{\infty}(n+1)(n+2)(n+3) \ldots(i+n-1) \chi_{\alpha}{ }^{n}=\frac{(n-1) !}{\left(1-\chi_{\alpha}\right)^{n}}
$$

Utilising linear combinations of equation 11, equations 8-10 simplify to equations 1214 respectively.

$$
\begin{aligned}
& \Gamma=\Lambda^{-1} \\
& \Gamma_{R}=\left(1-\chi_{C}\right) \Lambda^{-1} \\
& \Gamma_{S}=\left(1-\chi_{A}\right) \Lambda^{-1} \\
& \Lambda=1-\chi_{A}-\chi_{B}-\chi_{C}+\chi_{A} \chi_{C}
\end{aligned}
$$

It can be seen that the path expression products share a common denominator, $\Lambda$. As one of the PECs multiply every initial wave, the roots of $\Lambda$ can be used to find the conditions for resonance.

The amplitude and phase of the leftward and rightward propagating waves are found for each cavity by summing the initial waves and multiplying them by their respective PEC. For the reference cavity, the rightward and leftward wave amplitudes are denoted as $\alpha_{R}$ and $\beta_{R}$ respectively and are given in equations 16-17. In the following equations the first subscript of a variable refers to the cavity it is in reference to and the second subscript (where necessary) is in reference to the direction of travel. For 
example $F_{R L}$ is the amplitude coefficient of the leftward travelling wave originating from the excitation source within the reference cavity.

$$
\begin{aligned}
A_{R}=\Lambda^{-1}\{(1- & \left.\chi_{C}\right)\left(F_{R R} e^{-i \gamma x_{R}}+R_{R} F_{R L} e^{i \gamma x_{R}}\right) \\
& \left.+R_{R} T_{C}\left(F_{S L} e^{i \eta \gamma x_{S}}+R_{S} F_{S R} e^{i \gamma\left(2 a+\eta\left(2 b-x_{S}\right)\right)}\right)\right\} \\
B_{R}=\Lambda^{-1}\{(1- & \left.\chi_{C}\right)\left(F_{R L} e^{i \gamma x_{R}}+R_{C} F_{R R} e^{i \gamma\left(2 a-x_{R}\right)}\right) \\
& \left.+T_{C}\left(F_{R L} e^{i \eta \gamma x_{S}}+R_{S}\left[F_{S R} e^{i \eta \gamma x_{S}}+T_{C} F_{R R} e^{i \gamma x_{R}}\right] e^{i 2 \gamma(a+\eta b)}\right)\right\}
\end{aligned}
$$

Similarly, the rightward and leftward wave amplitudes for the sense cavity are given in equations 18-19 respectively.

$$
\begin{gathered}
A_{S}=\Lambda^{-1}\left\{\left(1-\chi_{A}\right)\left(F_{S R} e^{-i \eta \gamma x_{S}}+R_{C} F_{S L} e^{-i\left(2 \gamma a-\eta \gamma x_{S}\right.}\right)\right. \\
\left.+T_{C}\left(e^{i \gamma x_{R}}\left[F_{S R}+R_{R} F_{R L}\right]+R_{R} T_{C} F_{S L} e^{i \eta \gamma x_{S}}\right)\right\} \\
B_{S}=\Lambda^{-1}\left\{\left(1-\chi_{A}\right)\left(F_{S L} e^{i \eta \gamma x_{S}}+R_{S} F_{S R} e^{i \gamma\left(2 a+\eta\left(2 b-x_{S}\right)\right)}\right)\right. \\
\left.+R_{S} T_{C}\left(F_{R R}+R_{R} F_{R L}\right) e^{i \gamma\left(\left(2 a+x_{R}\right)+2 \eta b\right)}\right\}
\end{gathered}
$$

The displacement fields in each cavity are therefore described by equations 20 and 21 .

$$
\begin{aligned}
& \psi_{R}(x)=\left\{\begin{array}{cc}
\left|A_{R}+\right| B_{R}-F_{R L}\left|e^{-i\left(\gamma x_{R}+\angle\left(B_{R}-F_{A L}\right)\right)}\right| e^{i \gamma x} & x>x_{R} \\
\left|A_{R}+\right| B_{R}\left|e^{-i \angle B_{R}}-F_{R R} e^{-i \gamma x_{R}}\right| e^{i \gamma x} & x<x_{R}
\end{array}\right. \\
& \psi_{S}(x)=\left\{\begin{array}{cc}
\left|A_{S}+\right| B_{S}-F_{S L}\left|e^{-i\left(\eta \gamma x_{S}+\angle\left(B_{S}-F_{S L}\right)\right)}\right| e^{i \eta \gamma x} & x>x_{S} \\
\left|A_{S}+\right| B_{S}\left|e^{-i \angle B_{S}}-\right| F_{S R}\left|e^{-i \eta \gamma x_{S}}\right| e^{i \eta \gamma x} & x<x_{S}
\end{array}\right.
\end{aligned}
$$

The transmission and reflection coefficients of the arrays can be been found from the transfer matrix method presented in references [9] and [11] for the wave equation and Rayleigh waves in Lithium Niobate respectively. For comparison to the FEM model, the closed form expressions for the reflection and transmission amplitude across an $\mathrm{N}$-cell array from reference [9] are non-dimensionalised and shown in equations 2227. A negative phase shift equal to half the period in the cavity is added to define the termination of the cavity at the end of the last plated region, as defined in the model.

$$
\widetilde{T_{N}}=\frac{1}{\left(\widetilde{w} e^{-i \pi \widetilde{\Omega}} U_{N-1}(\tilde{\zeta})-U_{N-2}(\tilde{\zeta})\right) e^{i \pi \mu \widetilde{\Omega}}}
$$




$$
\begin{aligned}
& \widetilde{R_{N}}=\frac{z U_{N-1}(\tilde{\zeta})}{\left(\widetilde{w} e^{-i \pi \widetilde{\Omega}} U_{N-1}(\tilde{\zeta})-U_{N-2}(\tilde{\zeta})\right) e^{i \pi \mu \widetilde{\Omega}}} \\
& \tilde{z}=-i \epsilon_{-} \operatorname{Sin}\left(\frac{\pi \mu \widetilde{\Omega}}{\widetilde{v_{p}}}\right) \\
& \widetilde{w}=\left(\operatorname{Cos}\left(\frac{\pi \mu \widetilde{\Omega}}{\widetilde{v_{p}}}\right)-i \epsilon_{+} \operatorname{Sin}\left(\frac{\pi \mu \widetilde{\Omega}}{\widetilde{v_{p}}}\right)\right) e^{i \pi \mu \widetilde{\Omega}} \\
& \tilde{\zeta}=\operatorname{Re}(\widetilde{w}) \operatorname{Cos}(\pi \widetilde{\Omega})+\operatorname{Im}(\widetilde{w}) \operatorname{Sin}(\pi \widetilde{\Omega}) \\
& \epsilon_{ \pm}=\frac{1}{2}\left(\widetilde{v_{p}} \pm \frac{1}{\widetilde{v_{p}}}\right)
\end{aligned}
$$

Where $U_{N}(\tilde{\zeta})$ is the Nth Chebyshev polynomial of the second kind and $\widetilde{w}$ and $\tilde{z}$ are the non-dimensional form of the upper and lower left components of the single cell transfer matrix, respectively. The non-dimensional parameters used are defined in equations 28-31.

$$
\begin{gathered}
\widetilde{\Omega}=\frac{2 s f}{v_{0}} \\
\tilde{s}=\frac{s f}{v_{0}}
\end{gathered}
$$

$$
\begin{gathered}
\widetilde{v_{p}}=\frac{v_{p}}{v_{0}} \\
\mu=\frac{d}{s}
\end{gathered}
$$

Where $f$ is the operating frequency, $v_{0}$ is the substrate wave-speed, $v_{p}$ is the wavespeed within the plated regions. $s$ is the period within the array and $d$ is the length of the un-plated region per period, $\mu$ is therefore the plating ratio.

The relationship between the period within the array and the incident wavelength, $\lambda_{i}$, is therefore $\lambda_{i}=2 s / \widetilde{\Omega}$.

\subsection{Finite element model}

One of the main motivators for constructing a valid analytical solution for the SAW device was that it is an extremely difficult problem to solve using FEM. Firstly, due to the nature of the device utilising a break in the symmetry of the problem, the geometry cannot be reduced significantly by exploiting symmetric planes in the model. Additionally, in order to reduce bulk scattering and from the edges of the 
reflectors, it is necessary to ensure their thickness is sufficiently small when compared to the wavelength [11]. This leads to a large array of reflectors and consequently a model that is very large in comparison to the wavelength, likely on the order of hundreds of wavelengths long. Furthermore, a high stress point appears at every discontinuity in the surface, requiring a high mesh resolution to resolve it accurately. This requires mesh elements that are thousands of times smaller than the wavelength of interest. Thus the model is necessarily very large and involves high stress gradients and therefore is computationally expensive.

To validate the solution of section 2.1 it will be compared to an equivalent onedimensional acoustic problem that is readily solved using FEA. This amounts to a steady-state solution of an inhomogeneous one-dimensional Helmholtz equation with variable parameters. In this case the variable parameter is the wave-speed, which is perturbed periodically in the arrays as well as uniformly across the sense cavity. This model will serve to validate the derived displacement field expressions, with the use of the one-dimensional wave equation transfer matrix derived in references $[9,10]$. The solution of section 2.1 can then be used with confidence to describe SAW devices when used with the SAW transfer matrix solution of reference [11].

The geometry of the finite element model was simply a line split into sections reflecting the geometry shown in figure 2 . In the frequency domain, each section was governed by equation 32 . The parameter $c$ was set equal to unity for all sections. The definition of the parameter ' $a$ ' is shown in equation 33 where $\Omega$ is the excitation frequency and $v$ is the section wave-speed, which varied between sections according to figure 2 .

$$
\begin{aligned}
& \nabla \cdot(-c \nabla u(x))+\mathrm{a} u(x)=0 \\
& \mathrm{a}=-\frac{\Omega^{2}}{v^{2}}
\end{aligned}
$$

The length of the cavities was set to four wavelengths and the structure was terminated one wavelength after the enclosing arrays. Radiating boundary conditions were imposed at the terminations of the structure, as had been assumed in the analytical model. An excitation source was located in the centre of each array; a dipole acoustic source was selected which radiates two sinusoids of opposite phase in 
opposite directions, the amplitudes of which were set to unity. The numerical parameters were found from the non-dimensional parameters (equations 28-31) with $s$ and $v_{1}$ also set equal to unity.

\subsection{Contrast of analytical and numerical solutions}

Comparison to the FEM model is presented here as a means of validation of the model derived in section 3.4. In order to include all of the terms in the model, it will include excitation in both cavities. The comparison parameter will be the cavity amplitudes. This was evaluated from the FEM output as the maximum value within the displacement vector output from the respective cavity. The cavity amplitudes will be compared in terms of frequency response and variation of parameters $\eta, x_{R}, x_{S}, a$ and $\widetilde{v_{p}}$. Due to both models using identical BC's and forcing conditions and the simplicity of the numerical model, exact agreement is anticipated. The frequency response and parameter dependence plots are shown in figure 4 . The plots depict the expected exact agreement between the two models.
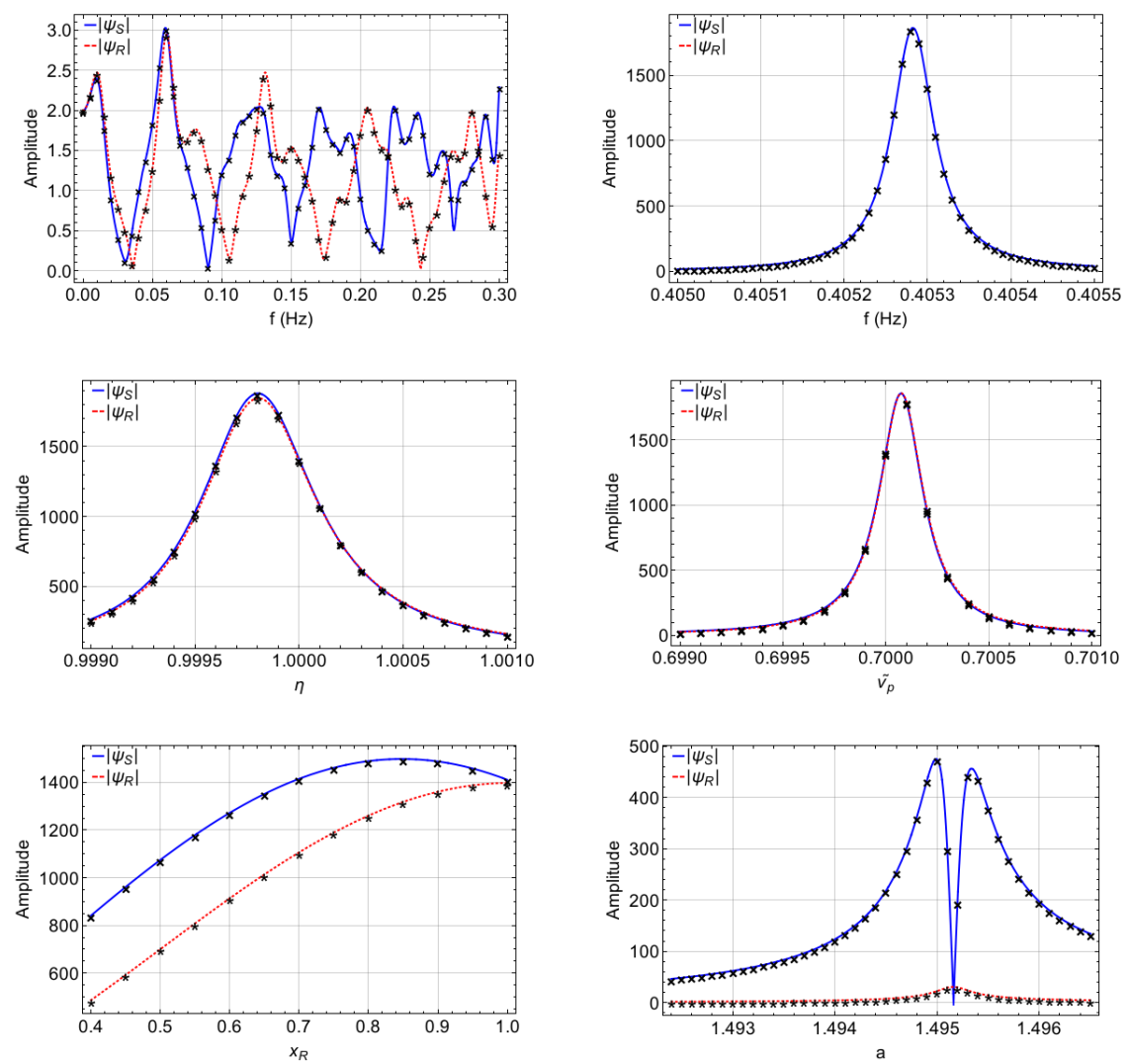

Figure 4 Validation plots showing cavity amplitudes expanded in various parameters. The full blue line depicts the sense cavity displacement amplitude and the dotted red line is that of the reference cavity. The plots markers represent the data points from the FEM model. 


\section{Analysis}

\subsection{Frequency response}

Resonance will occur when the absolute value of the denominator of equations 19 and 20 reach a minimum. For a non-dissipative system the minima will be equal to zero. Setting the outer reflection coefficients equal to negative one replicates a hard boundary condition, full reflection with a $\pi$ radian phase shift, eliminating the sole form of dissipation from the system. In this case the condition for resonance yields the systems natural frequencies and can be written explicitly as in equation 34.

$$
1+R_{C} e^{i 2 \pi \widetilde{\Omega} a}+R_{C} e^{i 2 \pi \widetilde{\Omega} \eta b}+\left({R_{C}}^{2}-T_{C}{ }^{2}\right) e^{i 2 \pi \widetilde{\Omega}(a+\eta b)}=0
$$

The numerators of equations 20 and 21 bear the initial waves. This dictates the effect of the forcing on the response and can be used to strategically superpose wave amplitudes and appear to cancel or amplify resonances. However, solely the denominator governs the total absolute value of displacement in the cavity, which could also be thought of as the total energy remaining within the cavity. The plot in figure 5 overlays $\Lambda^{-1}$ on the transmission spectrum of the coupling array. This demonstrates how the frequency response of the device varies within the pass and stop bands.

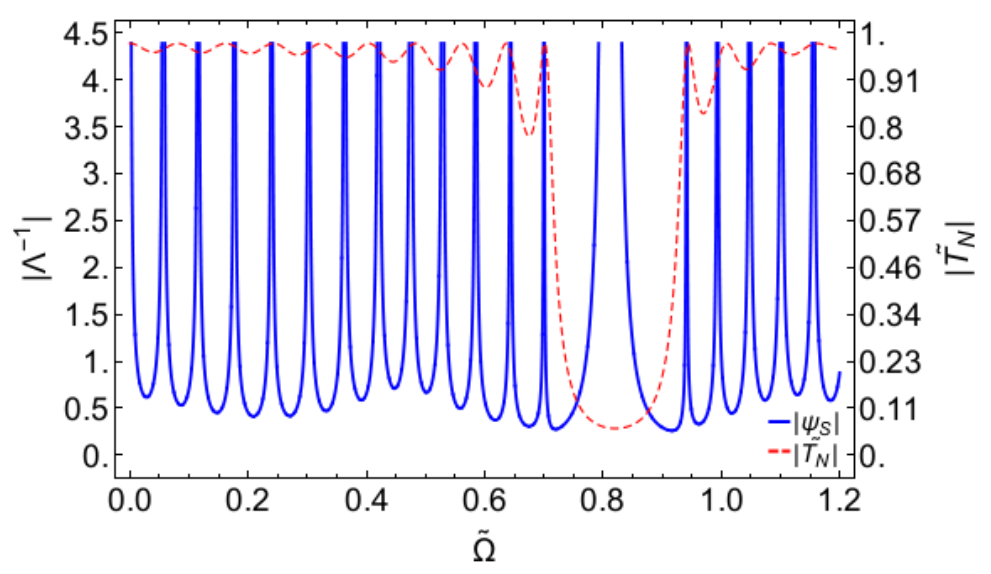

Figure 5 Frequency response and transmission spectrum 


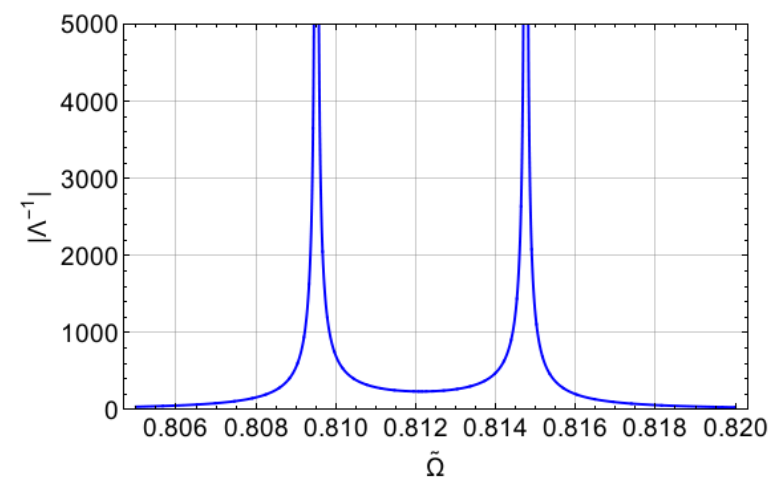

Figure 6 Frequency response within the stop band

It can be seen that the majority of the modes appear within the pass band. However, the higher resolution plot of figure 6 shows that two modes reside within the stop band, these are the analogues of the in-phase and anti-phase modes of the 2-DOF system described in section 1.1. This is demonstrated by the amplitude and phase plots of figure 7.
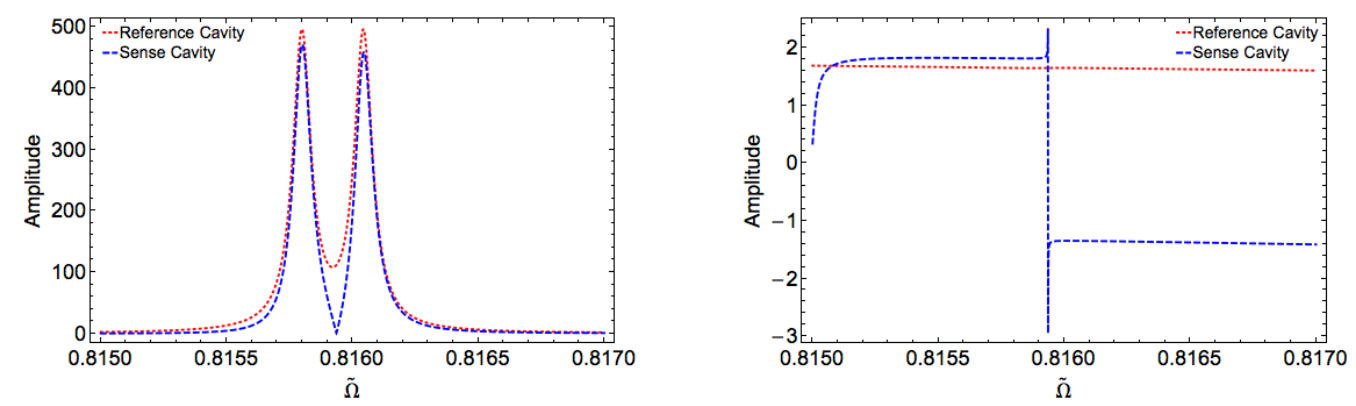

Figure 7. Amplitude (left) and phase (right) response of the two cavities. Matched amplitudes and opposite phases demonstrate the lower mode is the in-phase mode and the higher frequency mode the anti-phase mode.

\subsection{Qualitative description of device operation}

Localization is induced within the mode by altering the parameter $\eta$, the wave-speed ratio, shown explicitly in equation 35 .

$$
\eta=\frac{\gamma_{S E N S E}}{\gamma_{0}}=\frac{c_{0}}{c_{S E N S E}}
$$

The desired behaviour can be seen qualitatively from the plots of figure 8 which overlay the cavity amplitudes. It can be seen that in the symmetrical case $(\eta=1)$ the cavity amplitudes are well matched in both modes. As the system moves away from this condition, in either direction, localization is induced in both modes. 

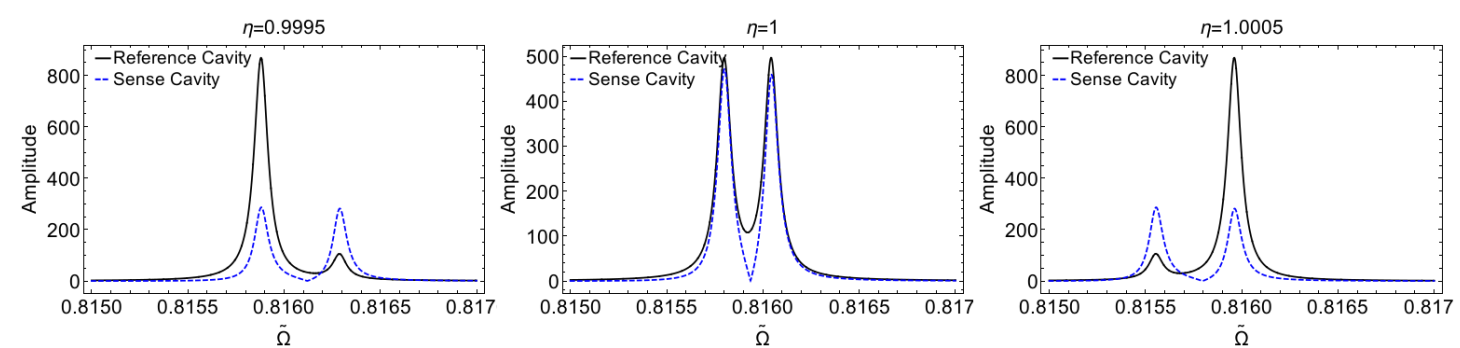

Figure 8. Amplitude localization demonstrated within both modes with the introduction of a disorder.

The modes can be tracked in frequency space by solving equation 34 for $\widetilde{\Omega}$.

Substituting this into the cavity amplitude expressions (equations 20-21) allows for calculation of the change in cavity amplitudes with varying parameters.

Figure 9 displays plots of both the cavity amplitudes and the amplitude ratio for the in-phase mode. Both the number of periods in the coupling array and $\eta$ were varied.

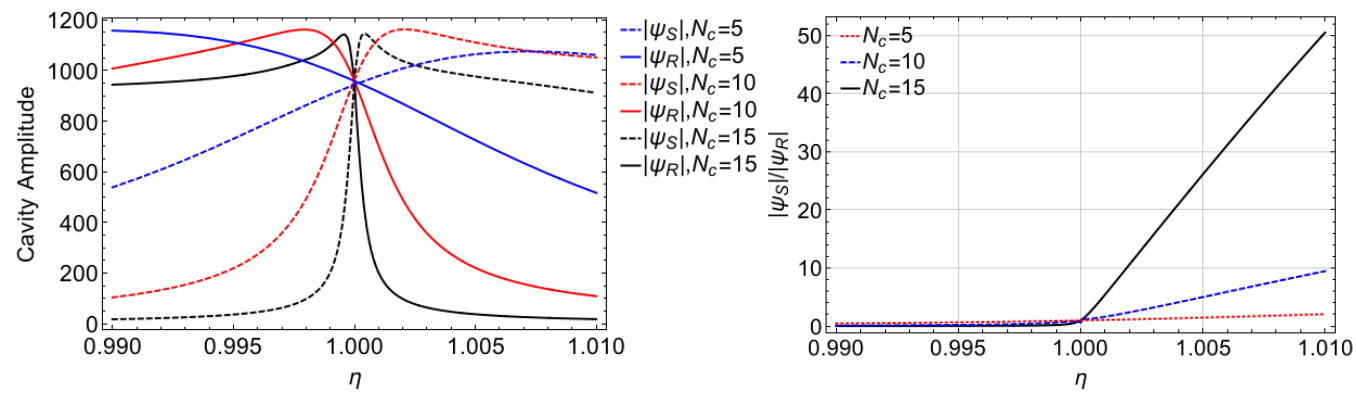

Figure 9. Cavity amplitudes (left) and amplitude ratio (right) for varying $\eta$ and coupling array periods

The point of intersection of all cavity amplitudes also lies of the $\eta=1$ line, this point represents an amplitude ratio of unity. The amplitude ratio plot demonstrates the expected device response when utilised as a sensor, which is an increase in amplitude ratio in proportion to the size of the disorder. The behaviour is identical for increasing and decreasing $\eta$, however, the amplitude ratio is inverted as the $\eta=1$ line is crossed. It is evident that increasing the number of periods in the coupling array leads to an increase in sensitivity. This is plotted directly in figure 10, for a fixed value of $\eta$. It is shown that the sensitivity increases exponentially as the number of periods in the coupling array is increased. 

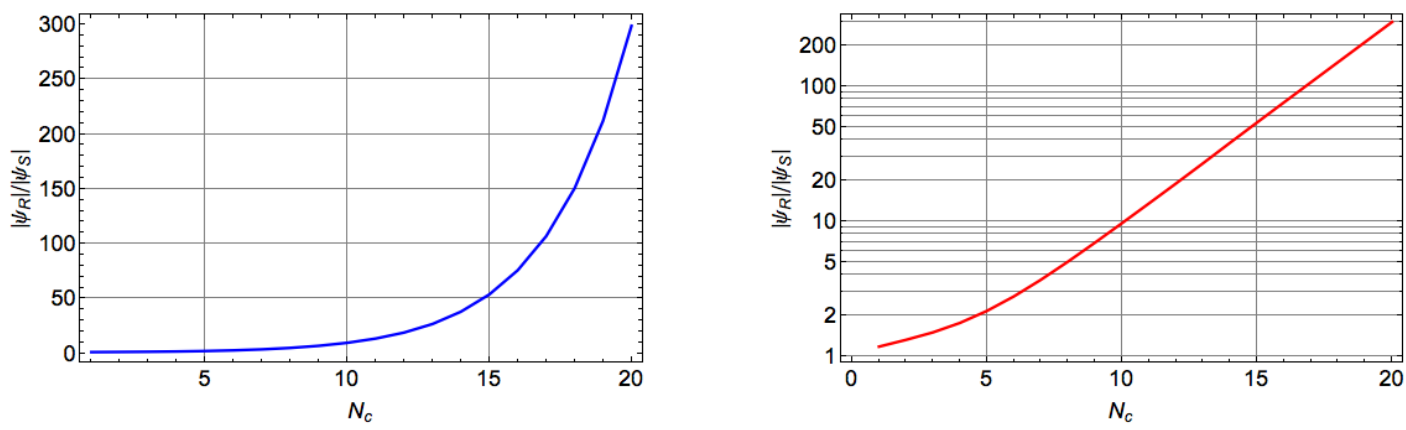

Figure 10 Amplitude ratio against couple array periods. Plotted on both a linear (left) and logarithmic (right) scale.

The full displacement field solution can be found from the FEM model, this is plotted in figure 11 for the in-phase mode demonstrating the change in the mode shape after the asymmetry is introduced.

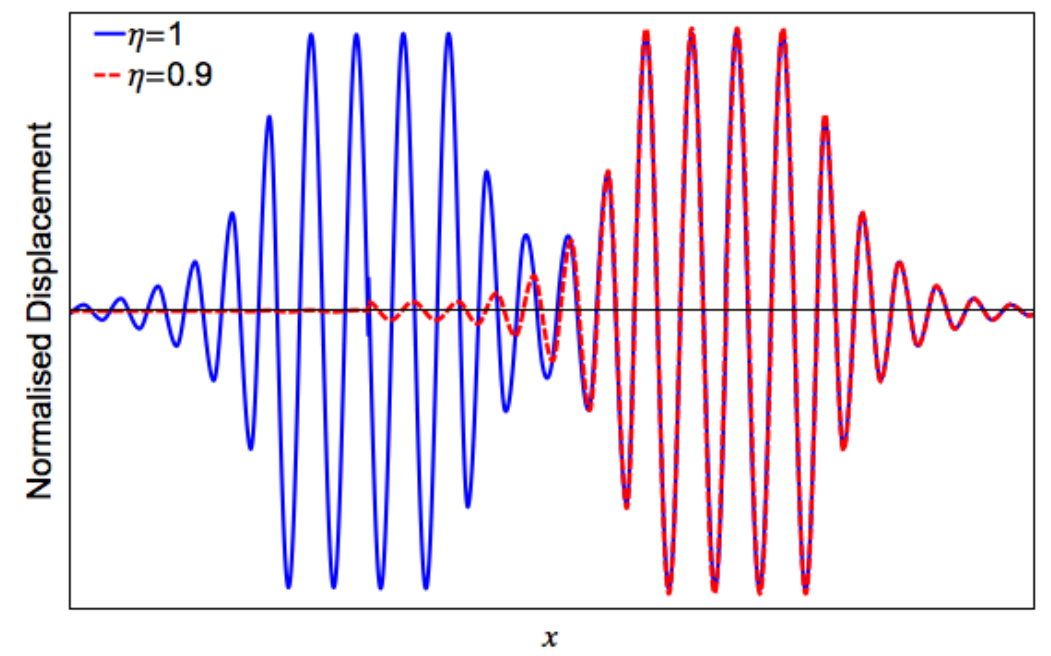

Figure 11. Full field solution demonstrating localization after the introduction of a disorder.

\section{Conclusions}

The principles of a novel SAW mode localization sensor have been outlined and has been found to behave similarly to the two degree-of-freedom examples described in references [2-5]. A general model has been presented that has shown exact agreement with simulated results from commercial FEM software. This analytical model provides an accurate tool for design and optimisation of the SAW mode localization sensor, accounting for the primary design and tuning parameters. 
The analysis has reported analogous operation to previous examples of mode localization sensors described in the literature. Additionally, it has been shown that the number of periods in the coupling array can be used to enhance the sensitivity of the device.

\section{References}

[1] L. Nicu, C. Bergaud, Modeling of a tuning fork biosensor based on the excitation of one particular resonance mode, J. Micromechanics Microengineering. 14 (2004) 727-736. doi:10.1088/0960-1317/14/5/011.

[2] M. Spletzer, A. Raman, A.Q. Wu, X. Xu, R. Reifenberger, Ultrasensitive mass sensing using mode localization in coupled microcantilevers, Appl. Phys. Lett. 88 (2006) 254102. doi:10.1063/1.2216889.

[3] M. Spletzer, A. Raman, H. Sumali, J.P. Sullivan, Highly sensitive mass detection and identification using vibration localization in coupled microcantilever arrays, Appl. Phys. Lett. 92 (2008) 114102. doi:10.1063/1.2899634.

[4] P. Thiruvenkatanathan, J. Yan, J. Woodhouse, a. Aziz, a. a. Seshia, Ultrasensitive mode-localized mass sensor with electrically tunable parametric sensitivity, Appl. Phys. Lett. 96 (2010) 081913. doi:10.1063/1.3315877.

[5] P. Thiruvenkatanathan, J. Woodhouse, J. Yan, a. a. Seshia, Limits to modelocalized sensing using micro- and nanomechanical resonator arrays, J. Appl. Phys. 109 (2011) 104903. doi:10.1063/1.3590143.

[6] T.E. Parker, G.K. Montress, Precision Surface-Acoustic-Wave (SAW) Oscillators, IEEE Trans. Ultrason. Ferroelectr. Freq. Control. 35 (1988) $342-$ 364. doi:10.1109/58.20455.

[7] T.H. Hanley, B.J. Gallacher, H.T.D. Grigg, Towards the exploitation of local resonances for novel MEMS devices, in: J. Awrejcewicz, M. Kazmierczak, J. Mrozowski, P. Olejnik (Eds.), Dyn. Syst. - Mechatronics Life Sci., 2015: pp. 207-216.

[8] N.S. Clarke, J.S. Burdess, A Rayleigh Resonator, Wave Motion. 13 (1991) 193-200.

[9] D.J. Griffiths, C. a. Steinke, Waves in locally periodic media, Am. J. Phys. 69 (2001) 137. doi:10.1119/1.1308266.

[10] P. Pereyra, E. Castillo, Theory of finite periodic systems: General expressions and various simple and illustrative examples, Phys. Rev. B. 65 (2002) 205120. doi:10.1103/PhysRevB.65.205120.

[11] B.K. Sinha, H.F. Tiersten, Variational analysis of the reflection of surface waves by arrays of reflecting strips, J. Appl. Phys. 47 (1976) 2824-2832. doi:10.1063/1.323079.

[12] K.F. Graff, Wave motion in elastic solids, 1991.

[13] J.D. Achenbach, Wave Propagation in Elastic Solids, 1975. doi:10.1016/B9780-7204-0325-1.50015-1. 
[14] D.M. Mead, Wave Propagation in Continuous Periodic Structures: Research Contributions From Southampton, 1964-1995, J. Sound Vib. 190 (1996) 495524. doi:10.1006/jsvi.1996.0076. 